\title{
Quantifying the degradation of degradable implants and bone formation in the femoral condyle using micro-CT 3D reconstruction
}

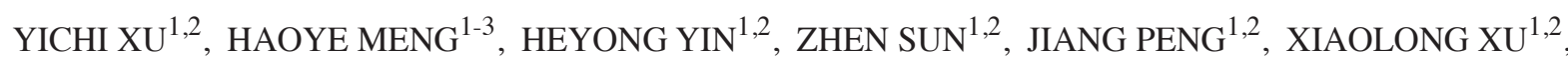 \\ QUANYI GUO ${ }^{1,2}$, WENJING XU ${ }^{1,2}$, XIAOMING YU ${ }^{1,2}$, ZHIGUO YUAN $^{1,2}$, BO XIAO $^{1,2}$, CHENG WANG $^{1,2}$, \\ YU WANG ${ }^{1,2}$, SHUYUN LIU ${ }^{1,2}$, SHIBI LU ${ }^{1,2}$, ZHAOXU WANG $^{4}$ and AIYUAN WANG ${ }^{1,2}$ \\ ${ }^{1}$ Institute of Orthopedics; ${ }^{2}$ Key Laboratory of Musculoskeletal Trauma and War Injuries, \\ Chinese PLA General Hospital, Beijing 100853; ${ }^{3}$ School of Materials Science and Engineering, University of \\ Science and Technology Beijing, Beijing 100083; ${ }^{4}$ Testing Department of Biomaterials and Tissue Engineering Products, \\ Chinese National Institutes for Food and Drug Control, Beijing 100050, P.R. China
}

Received September 30, 2016; Accepted August 31, 2017

DOI: $10.3892 / \mathrm{etm} .2017 .5389$

\begin{abstract}
Degradation limits the application of magnesium alloys, and evaluation methods for non-traumatic in vivo quantification of implant degradation and bone formation are imperfect. In the present study, a micro-arc-oxidized AZ31 magnesium alloy was used to evaluate the degradation of implants and new bone formation in 60 male New Zealand white rabbits. Degradation was monitored by weighing the implants prior to and following implantation, and by performing micro-computed tomography (CT) scans and histological analysis after $1,4,12,24,36$, and 48 weeks of implantation. The results indicated that the implants underwent slow degradation in the first 4 weeks, with negligible degradation in the first week, followed by significantly increased degradation during weeks 12-24 ( $\mathrm{P}<0.05)$, and continued degradation until the end of the 48 -week experimental period. The magnesium content decreased as the implant degraded $(\mathrm{P}<0.05)$; however, the density of the material exhibited almost no change. Micro-CT results also demonstrated that pin volume, pin mineral density, mean 'pin thickness', bone surface/bone volume and trabecular separation decreased over time $(\mathrm{P}<0.05)$, and that the pin surface area/pin volume, bone volume fraction, trabecular thickness, trabecular number and
\end{abstract}

Correspondence to: Professor Zhaoxu Wang, Testing Department of Biomaterials and Tissue Engineering Products, Chinese National Institutes for Food and Drug Control, 2 Tiantan Xili, Beijing 100050, P.R. China

E-mail: wangzx@nicbp.org.cn

Professor Aiyuan Wang, Institute of Orthopedics, Chinese PLA General Hospital, 28 Fuxing Road, Beijing 100853, P.R. China

E-mail: aiyuanwang301@126.com

Key words: micro-computed tomography, magnesium alloy, degradation, bone formation, in vivo tissue mineral density increased over time $(\mathrm{P}<0.05)$, indicating that the number of bones and density of new bone increased as magnesium degraded. These results support the positive effect of magnesium on osteogenesis. However, from the maximum inner diameter of the new bone loop and diameter of the pin in the same position, the magnesium alloy was not capable of creating sufficient bridges between the bones and biomaterials when there were preexisting gaps. Histological analyses indicated that there were no inflammatory responses around the implants. The results of the present study indicate that a micro-arc-oxidized AZ31 magnesium alloy is safe in vivo and efficiently degraded. Furthermore, the novel bone formation increased as the implant degraded. The findings concluded that micro-CT, which is useful for providing non-traumatic, in vivo, quantitative and precise data, has great value for exploring the degradation of implants and novel bone formation.

\section{Introduction}

Common implant materials, including titanium alloys, stainless steel and cobalt-chromium alloys, have considerable advantages in terms of their load-bearing capabilities and resistance to fatigue, wear and corrosion (1-3). However, these traditional materials also have multiple disadvantages. For instance, their mechanical properties differ from those of natural bone, which may cause stress shielding (4-6). A second surgery is often required to remove the implant after the tissues have healed, which markedly increases the risk and costs of healthcare $(7,8)$. Furthermore, implant materials release cytotoxic ions and may cause physical irritation due to their rigidity $(9,10)$.

Recently, magnesium and its alloys have attracted increasing interest as innovative biodegradable materials, particularly due to their potential use as temporary orthopedic implants $(11,12)$. The fundamental properties of magnesium make it relatively suitable for this application (13); it has low density and elastic modulus, both of which are close to that of natural bone, thus preventing stress shielding during fracture 
consolidation $(14,15)$. In addition, magnesium is biocompatible and essential for human metabolism as a cofactor for many enzymes (16). Notably, magnesium ions that are produced as a result of implant degradation have been reported to aid in the growth and healing of tissues $(17,18)$. Magnesium is well tolerated by the human body and does not induce systemic inflammatory reactions or negatively affect the cellular blood composition (19). It also degrades in aqueous solutions, and is prone to degradation in body fluids (20); thus, follow-up surgery to remove the implant is not required (21). Finally, due to its functional roles and presence in bone tissue, magnesium may exert stimulatory effects on the growth of new bone tissue (21-23).

Despite these advantageous properties, magnesium and its alloys have not been widely used as human body implants to date. A major drawback is that magnesium alloys tend to corrode rapidly in chloride solutions, including the physiological environment, leading to a loss in their mechanical integrity before their expected service life $(1,23,24)$. One method of altering the surface and degradation properties without adding a coating material, and thus inducing other potentially irritating materials, is micro-arc oxidation $(25,26)$. This method of surface modification, which is based on the principle of plasma-electrolytic oxidation, produces an increased oxide layer at the surface (27). Micro arc-treated magnesium surfaces exhibit improved resistance to corrosion in various environments $(28,29)$. In vitro tests in simulated body fluid have repeatedly confirmed the favorable behavior of surface-treated magnesium alloys regarding reduced dissolution and enhanced biocompatibility (30).

There has been much research on degradable implants for applications in orthopedics (31-34), such as those made of polylactic-co-glycolic acid and magnesium alloys. It has been reported that the degradation of implant material and formation of new bone, and notably, the balance between these two processes, are important evaluation indices (35). Traditional methods of assessing degradation have unavoidable disadvantages, and the evaluation of new bone formation generally relies on images obtained from histology and radiography, which are imprecise and difficult to analyze quantitatively (36,37). Thus, the present study used images and data from micro-computed tomography (micro-CT), as a non-traumatic, in vivo, quantitative and precise process $(38,39)$.

Over the past decade, the number of orthopedic studies that have used micro-CT imaging has increased (40-49). Higher spatial and temporal resolution are key technical advances that have enabled researchers to capture increasingly detailed anatomical images of small animals and monitor the progression of orthopedic disease in small animal models $(38,50)$. Furthermore, a range of data may be derived from micro-CT, including bone volume (BV), bone mineral content (BMC), bone mineral density (BMD), bone volume fraction (BVF), bone volume/tissue volume (BV/TV), bone surface/bone volume (BS/BV), trabecular thickness (Tb.Th), trabecular number (Tb.N) and trabecular separation (Tb.Sp). With the development of radiography methods for the evaluation of implants, measuring the weight loss of implants and histological analysis, as 'traumatic' methods, and micro-CT imaging, as a 'non-traumatic' method, have become mainstream (51); however, quantitative analyses and methods that evaluate the association between implant material degradation and new bone formation are still lacking $(52,53)$.

In the present study, micro-CT images and data were used to assess the degradation of micro arc-oxidized AZ31 magnesium alloy implants in vivo. Changes in the volume of the AZ31 implants were assessed, and the application of the degradable implant for bone-regeneration was evaluated. By applying micro-arc surface treatment, the initial intention was to decrease the degradation rate of the magnesium implants in the initial post-implantation period and reduce the impact of degradation products on the postoperatively irritated surrounding tissue, then to slow dissolution of the implant material once the implant's function became redundant. Generally, a cylinder of the same size and position of the pin was selected as the region of interest (ROI) to analyze the degradation of the material, and a new larger ROI in the same shape and position was selected to observe new bone formation and assess the stimulatory effects of magnesium alloys on bone growth.

\section{Materials and methods}

Implants. Micro arc-oxidized cylindrical pins $(\mathrm{n}=60$; diameter $2.0 \mathrm{~mm}$, length $6.0 \mathrm{~mm}$, weight $0.500 \mathrm{~g}$ ) made of AZ31 material were used (Trauson Medical Instrument Co., Ltd., Changzhou, China). AZ31 is a fast-degrading alloy of magnesium with 3 wt.\% Al, 1 wt.\% $\mathrm{Zn}$ and 0.15 wt.\% $\mathrm{Mn}$.

Experimental design. All animal experiments were conducted following ethical guidelines by Ethics Committee of Chinese PLA General Hospital (Beijing, China), obtained international standard authentication-SIDCER (54) and were authorized by the Institutes for Food and Drug Control of China and KEYU Animal Experiment Center.

A total of 60 male New Zealand white rabbits (body weight 2.2-2.5 kg, 3 months old) were purchased from KEYU Animal Experiment Center (Beijing, China; accreditation number SCXK(Jing) 2012-0004, certification no. 11400800001109) for the present study. Experiments were performed under standard conditions throughout the study (temperature, $23 \pm 2^{\circ} \mathrm{C}$; relative humidity, $60 \pm 10 \%$; with access to a 12-h light/dark cycle). Rabbits had been ensured adequate food and water. Each rabbit had an AZ31 pin implanted into its right femoral condyle. Rabbits were randomly divided into six groups $(n=10)$, and were sacrificed after 1, 4, 12, 24, 36 or 48 weeks (1 group per time-point). Each pin was weighed prior to implantation and following sacrifice. Micro-CT, 3D reconstruction and histological examinations were performed following sacrifice.

Surgical procedure. Rabbits were fasted for $12 \mathrm{~h}$ prior to surgery and then anesthetized with an intraperitoneal injection of 3\% sodium pentobarbital (Sigma-Aldrich; Merck KGaA; Darmstadt, Germany) with a dosage of $24 \mathrm{mg} / \mathrm{kg}$. Surgery was performed under sterile conditions. First, full-thickness lesions were created with $2.0 \mathrm{~mm}$ Kirschner pins (Beijing Fule Science \& Technology Development Co., Ltd., Beijing, China). AZ31 alloy cylindrical pins were implanted into the defects in the right femoral condyles. All pins were $\gamma$-ray-sterilized with $29 \mathrm{kGy}$ of ${ }^{60} \mathrm{Co}$ radiation prior to surgery. All rabbits received an intramuscular anti-inflammatory 
injection with penicillin (Sigma-Aldrich; Merck KGaA) with a dosage of 400,000 U/day following surgery and were housed individually. Postoperatively, the rabbits were allowed to move freely in their cages without external support and with unrestricted weight bearing. Daily clinical observations were made throughout the study period.

Weighing. Prior to implantation, the AZ31 pins were weighed on an electronic balance (accuracy, $\pm 0.001 \mathrm{~g}$ ). At 1, 4, 12, 24, 36 or 48 weeks, pins were removed from the femoral condyle and dry machined with clean tools. After machining, the pins were cleaned with pure ethanol in an ultrasonic bath and dried in warm air (23). The pins were weighed again and the difference between the pre- and post- implantation weights was calculated. To explore the difference between weight and Micro-CT methods, the pin weight fraction was calculated using the following formula: Weight loss of implanted pins/weight before implantation. Subsequently the pin weight fraction was compared with the pin volume fraction.

Micro-CT. Micro-CT is an emerging technology that permits non-invasive, tissue-preserving imaging and quantitative morphometry of bone structure in three dimensions (55-56). Scans were performed with an RS-9 micro-CT (GE Healthcare, Chicago, IL, USA) to assess the condyle before it was placed in $25 \%$ formic acid solution for decalcification. The micro-CT system was operated at $80 \mathrm{kV}$ tube voltage and $450 \mu \mathrm{A}$ tube electric current, with a scan resolution of $45 \mu \mathrm{m}$ and exposure time of $400 \mathrm{msec}$. Images were reconstructed and the pin volume, bone volume, BMD, tissue mineral density (TMD), BMC, BVF, BV/TV, BS/BV, Tb.Th, Tb.N, and Tb.Sp were analyzed using the built-in software (Version MicroView Advanced Bone Analysis Application 2.2; GE Healthcare) of the micro-CT equipment at each time point. A cylinder of the same size was selected from the corresponding region around the pins of the femoral condyle (Fig. 1A-F). A cylindrical ROI was set at each time point, matching the size of the magnesium alloy pins, $2.0 \mathrm{~mm}$ in diameter and $6.0 \mathrm{~mm}$ in length, ensuring that the ROI and pin fully overlapped (Fig. 1C). The threshold was set to 850 and the ROI was highlighted using the built-in software (Fig. 1D). The magnesium alloy was considered as bone tissue to assess degradation of the implant. Similar to the bone volume fraction, the Mg volume fraction was applied as: Degraded alloy pin volume/pin volume before implantation. The BMD and TMD reflected the $\mathrm{Mg}$ cylinder mineral and CT image densities, respectively. However, unlike natural bone, the pins had no trabecular, and thus the trabecular meant the thickness of the pin was calculated from the radius of its cross-section. To investigate new bone formation, the previous ROI was replaced with a larger ROI (diameter $2.5 \mathrm{~mm}$, length $6.5 \mathrm{~mm}$ ) in the same shape and position (Fig. 1E), from which new bone formation and the stimulatory effects on the growth of new tissue were observed. The threshold was then set at 1,000 and the ROI was highlighted with the built-in software (Fig. 1F). The maximum inner diameter of the new bone loop in the cross-section of the magnesium pin was selected, and the diameter of the pin was measured with the built-in software at the same position. Magnesium degradation and bone ingrowth were subsequently investigated.
Histological processing. The femoral condyles were excised and fixed in a $10 \%$ buffered neutral formalin solution for 1 week at room temperature at weeks 4, 24 and 48. Following decalcification, the samples were cut in half longitudinally and embedded in paraffin wax. Central sections ( $5 \mu \mathrm{m}$ thick) were cut from the femoral condyle with a Leitz 1512 microtome (Leica Microsystems GmbH, Wetzlar, Germany) and stained at room temperature for $10 \mathrm{~min}$ with hematoxylin and eosin (H\&E) for histological examination. Microscopic images were captured using a light microscope (dotSlide Virtual Slide System; Olympus Corporation, Tokyo, Japan) at x40, x100 and x200 magnification.

Statistical analysis. Statistical analyses were performed using SPSS software (version 22.0; IBM Corp., Armonk, NY USA). Experimental values were expressed as the mean \pm standard deviation and were analyzed using an unpaired Student's t-test to determine differences between the pre-implantation value and the post-implantation values at each time point. $\mathrm{P}<0.05$ was considered to indicate a statistically significant difference.

\section{Results}

Changes in the weight of implant material. According to the weight change of pins at each time point (Fig. 2), there was almost no weight loss of the implants within the first week (-0.007 g). However, by week 12, significant weight loss of the pins was observed compared with their pre-implantation weights (-0.017 g; P<0.05; Fig. 2). During weeks 12-24, the rate of weight loss was markedly increased when compared with weeks 1-4 and 4-12. After week 24, the rate of weight loss gradually decreased. This may be due to the production of new bone during corrosion of the micro arc-oxidized surface at the later time points.

Micro-CT evaluation of implant degradation. The micro-CT images in Fig. 3 provide examples of the degradation process of an AZ31 implant at each time point. In the first week of the first month, little corrosion was observed, and the boundary of the pin was continuous and smooth (Fig. 3A). By week 4, the surface of the pin appeared blurred (Fig. 3B), indicating that the rate of degradation had increased compared with week 1 . Corrosion pitting became evident at week 12 (Fig. 3C), and during weeks 12-24 the surfaces of the pins were surrounded by new bone tissue (Fig. 3C and D). These results suggest that degradation of the implants accelerated between weeks 4-24 and was markedly faster than that in the first 4 weeks. From week 36 , corrosion pitting became more obvious, the boundary of the pin was inconclusive and the corners of the pins became indistinct (Fig. 3E and F). The images at this point suggested that degradation was still proceeding and the area of corrosion pitting was expanding. The acceleration of the degradation rate was observed at the preexisting corrosion points, and there did not appear to be corrosion at new positions on the surface of the pins. As indicated in Fig. 3F, corrosion pitting was evident on all surfaces and degradation was clear by week 48 . However, the micro-CT images also identified a small amount of hydrogen gas around the pins at weeks 4, 12 and 48 only (Fig. 3B, C and F), however, no apparent trend was observed. 

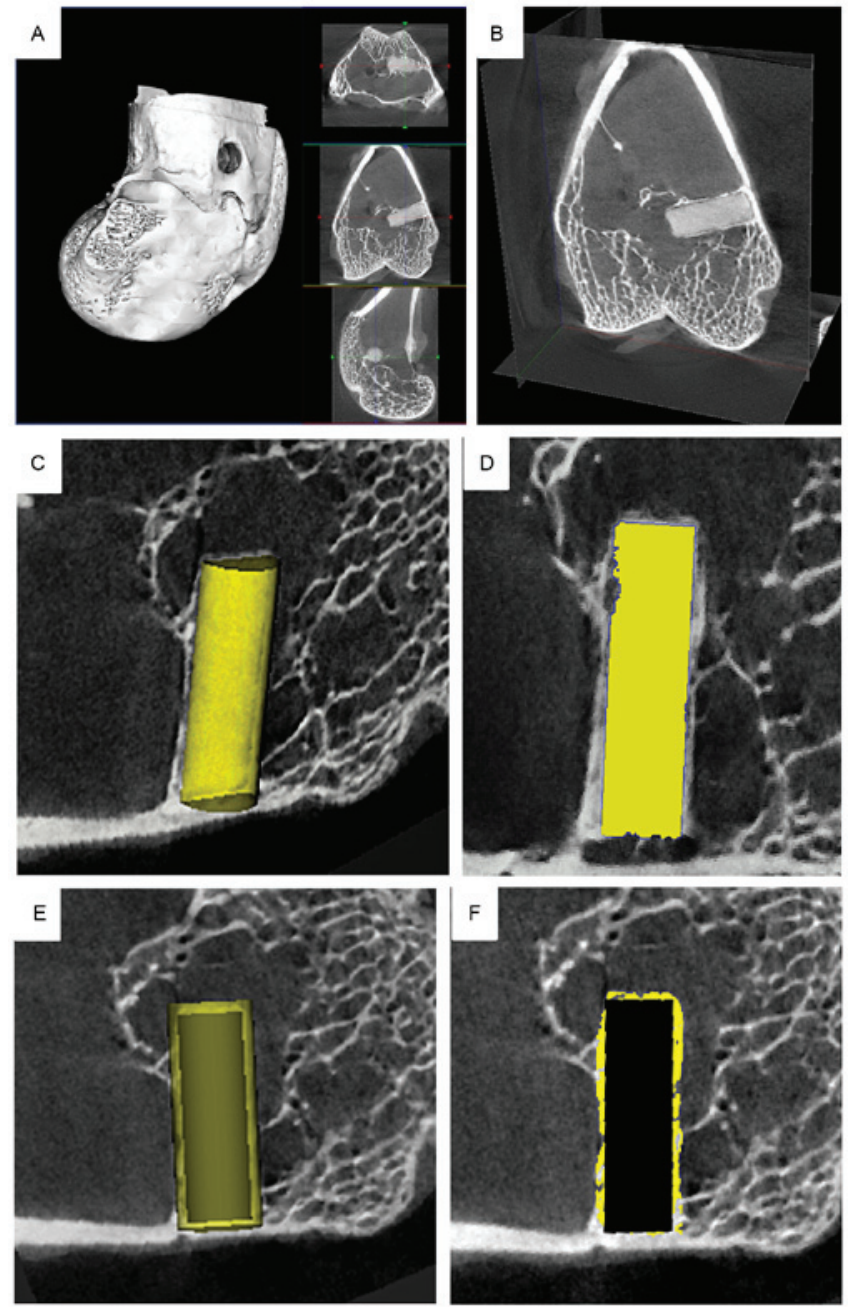

Figure 1. Micro-computed tomography images and 3D reconstruction of the femoral condyle. (A) 3D reconstruction of the femoral condyle and (B) longitudinal section in the 3D coordinate. (C) ROI (diameter $=2.0$ and length $=6.0 \mathrm{~mm}$ ) and (D) highlight of ROI of the magnesium-alloy pin. (E) New ROI (diameter $=2.5$ and length $=6.5 \mathrm{~mm}$ ) and $(\mathrm{F})$ highlight of ROI of bone formation around the pin after blanking the previous ROI. ROI, region of interest.

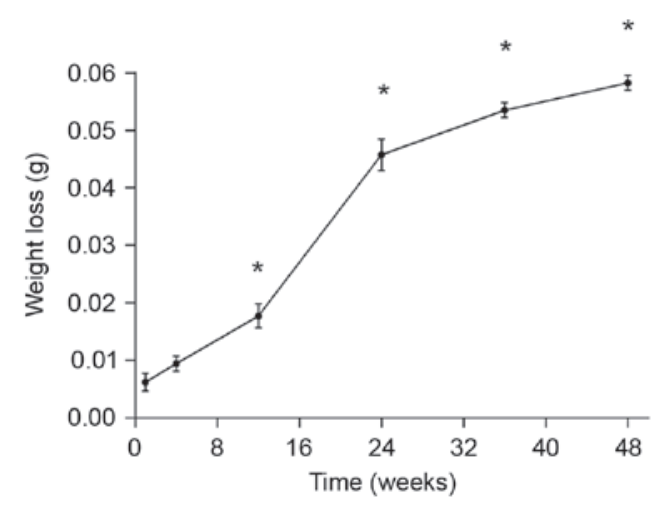

Figure 2. In vivo weight loss of implanted AZ31 pins during the study period. ${ }^{*} \mathrm{P}<0.05$ vs. prior to implantation.

Similar to the micro-CT images, the data exported from the built-in software of the micro-CT equipment provided precise results (Fig. 4). The pin volume fraction was $\geq 99.0 \%$ during the first month of the study, indicating that degradation

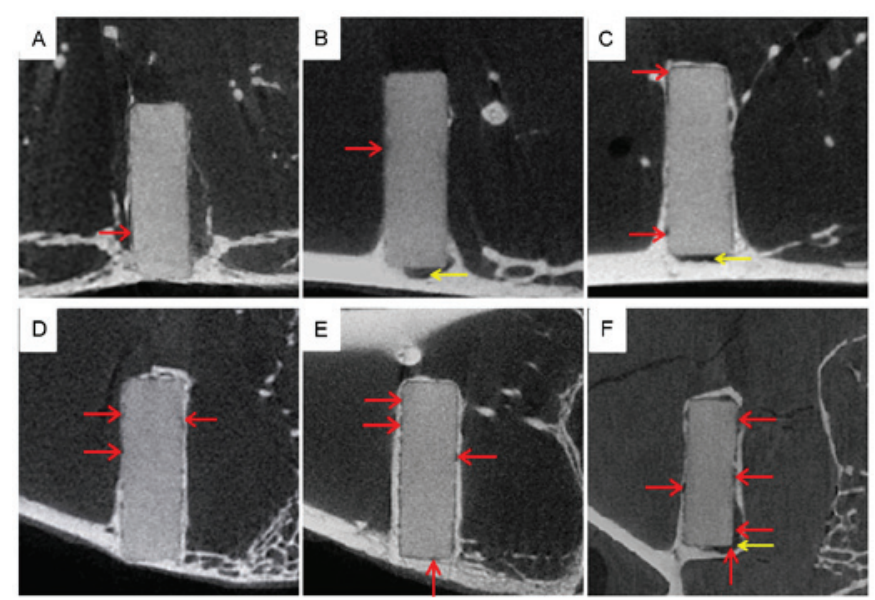

Figure 3. Region of interest and degradation of the magnesium alloy pins at (A) 1, (B) 4, (C) 12, (D) 24, (E) 36 and (F) 48 weeks. The pins degraded over time at different rates over the course of the experiment. Red arrows indicate corrosion pitting. Yellow arrows indicated hydrogen gas.

was slow and minor, and remained above $96.5 \%$ until week 12 , after which the volume fractions were significantly lower when compared with the pre-implantation value $(\mathrm{P}<0.05$; Fig. $4 \mathrm{~A})$. From week 12 to 24 , the pin volume fraction decreased markedly faster than in the first 3 months, and reached $91.2 \%$ by week 24. During this period, the micro arc-oxidized surface was being degraded and new bone tissue had not yet been sufficiently produced (Fig. 3A-D). After week 24, the rate of decrease slowed, and during the final 24 weeks, pin volume fraction only decreased by $2.25 \%$ (Fig. 4A). This may have been due to enclosure of the residual pin by new bone tissue, thus reducing contact between the metal and tissue fluid.

The AZ31 magnesium alloy pin was regarded as bone to analyze the BMD and TMD, which reflected the Mg cylinder mineral and CT image densities, respectively. The Mg cylinder mineral density continuously decreased throughout the study period, degrading most rapidly between weeks 12 and 24. From week 12 , the $\mathrm{Mg}$ cylinder material densities were significantly decreased compared with the pre-implantation value $(\mathrm{P}<0.05$; Fig. 4B), and by week 48 it had reached $403.1424 \mathrm{mg} / \mathrm{cc}$. By contrast, no significant change was observed in the pin CT image density, which started at 683.6439 (week 1) and ended at $644.9468 \mathrm{mg} / \mathrm{cc}$ (week 48; Fig. 4B). These results indicate that $\mathrm{Mg}$ content decreased as the implant degraded, while the density of the material underwent little change.

During degradation, the volume of the pin decreased, most notably during weeks 12-24 (Fig. 4A). Furthermore, with corrosion pitting, the surface of the pins became rough (Fig. 3C-F), which may increase the superficial area. Thus, the ratio between the surface area and volume of the pins increased throughout the study period, and were significantly higher compared with the pre-implantation value from week $24(\mathrm{P}<0.05$; Fig. $4 \mathrm{C})$. However, the surface area of the pins was preserved during the first 4 weeks (Fig. 4C). Unlike natural bone, the pins had no trabecular, and thus the trabecular mean thickness of the pin was calculated from the radius of its cross-section, and theoretically should be $1.0 \mathrm{~mm}$. The computer creates a theoretical line that automatically runs through the longitudinal axis of the AZ31 pin, and the radius of its cross-section represents 

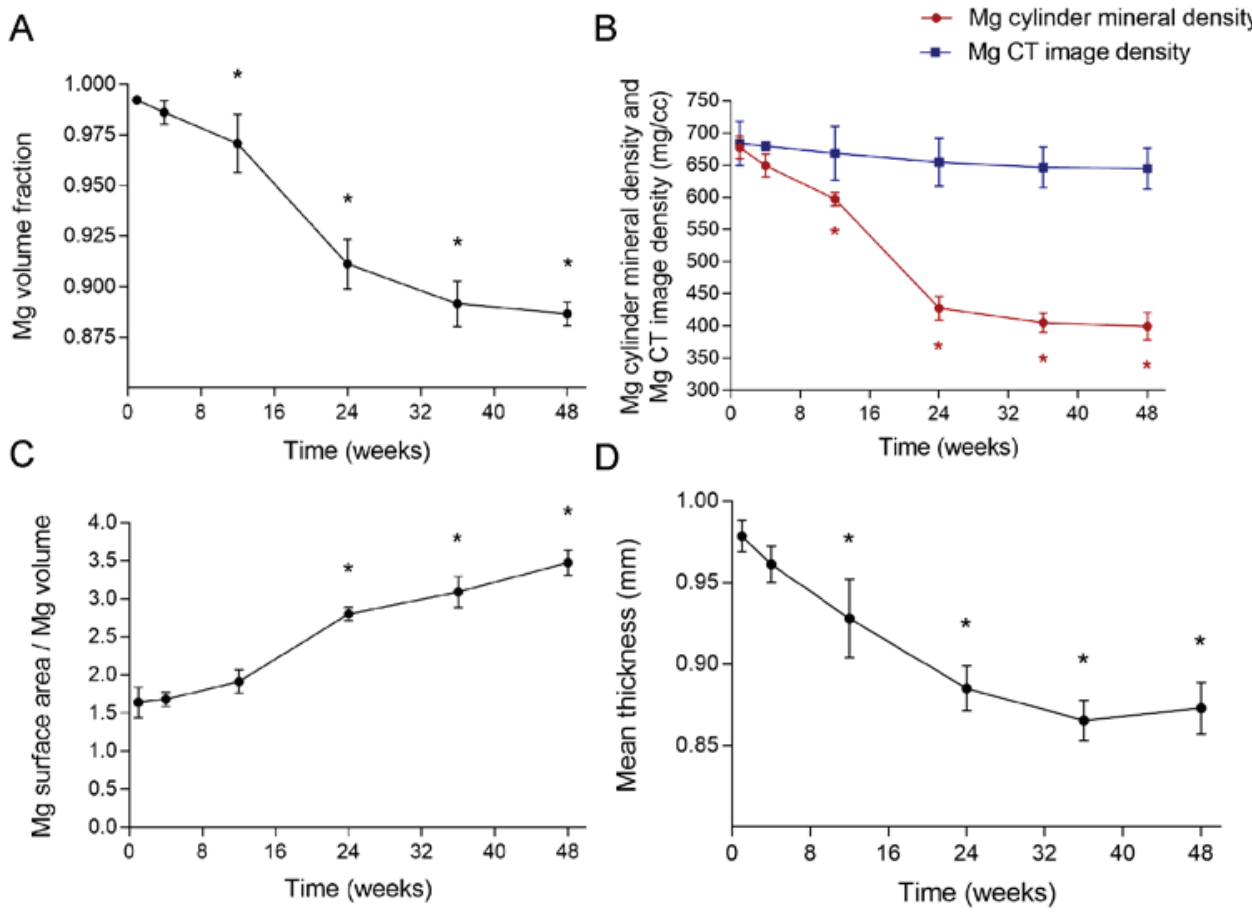

Figure 4. In vivo degradation of implanted AZ31 pins during the study period, assessed via micro-CT measurements. (A) Pin volume fraction. (B) Alloy cylinder mineral density and CT image density. (C) Ratio of the surface area and volume. (D) Mean thickness of the pin. CT, computed tomography. ${ }^{*}<0.05$ vs. prior to implantation.

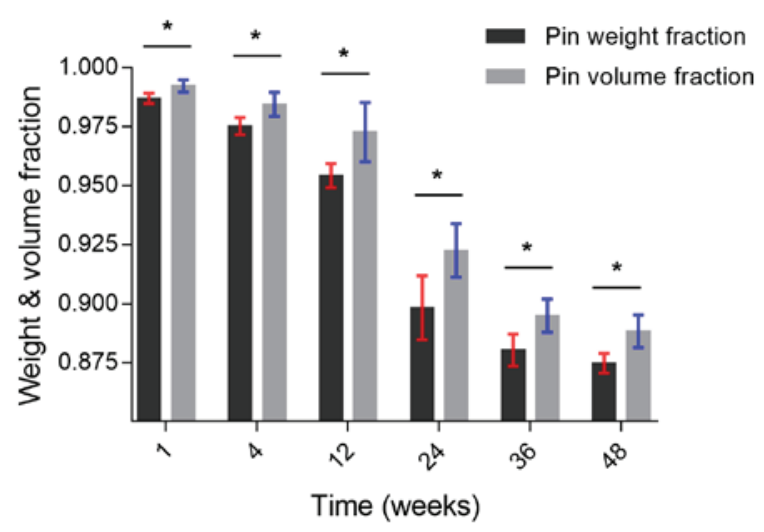

Figure 5. Weight fraction and volume fraction of the AZ31 magnesium alloy pins. ${ }^{*} \mathrm{P}<0.05$ vs. prior to implantation.

the pin's mean thickness. During the period investigated, degradation of the pins and increased corrosion pitting on the surface resulted in a decrease in the pin radius. The thickness of the pre-implantation pin was $1.0 \mathrm{~mm}$ and remained above $0.925 \mathrm{~mm}$ up to week 12 , from which point it was significantly decreased compared with the pre-implantation value $(\mathrm{P}<0.05$; Fig. 4D). During weeks 24-36, the thickness decreased more slowly than in 1-24 weeks, and during weeks 36-48, a slight increase in pin thickness was observed. This may have been due to the stimulatory effects of magnesium on the growth of new bone tissue at the metal corrosion loci.

To analyze pin degradation, the changes in pin weight fraction and volume fraction over the 48 weeks was plotted (Fig. 5). The results of two methods used (weighing and Micro-CT) to

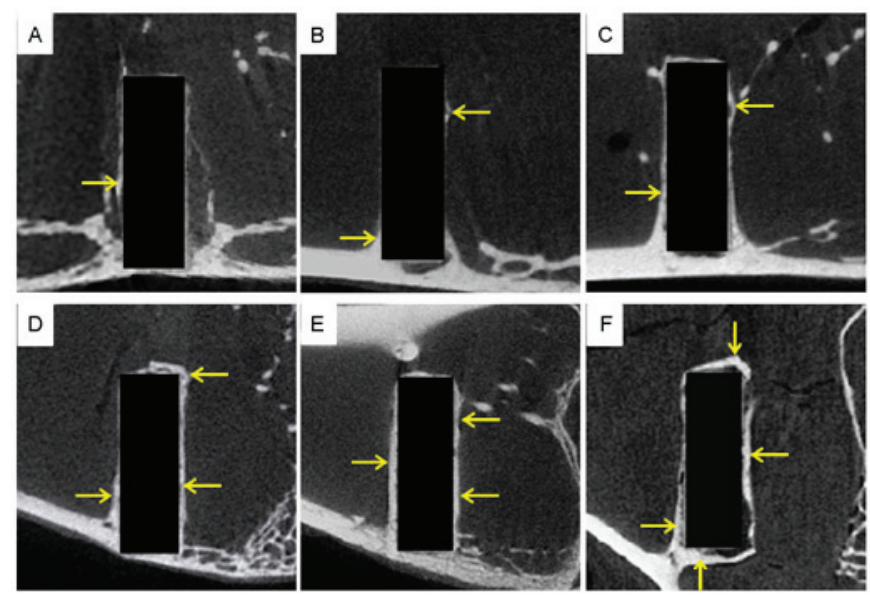

Figure 6. After blanking the previous ROI, a new larger ROI was selected (diameter $=2.5$ and length $=6.5 \mathrm{~mm}$ ) in the same shape and same position to observe new bone formation and assess the stimulatory effects of magnesium alloy on the growth of new bone tissue. New bone formed over time at different rates throughout the experiment. Representative micro-computed tomography images from (A) 1, (B) 4, (C) 12, (D) 24, (E) 36 and (F) 48 weeks are shown. Yellow arrows indicate new bone.

calculate amount of remaining pin were similar: both the weight and volume of the pins decreased over the 48 weeks, and during weeks 12-24, the decrease of the weight and volume of the pin was markedly fast. However, the data were not wholly consistent: at each time point, the quantity of magnesium corrosion by weight was significantly greater than by volume $(\mathrm{P}<0.05$; Fig. 5$)$.

Micro-CT evaluation of bone formation. A new, larger ROI was subsequently selected in the same shape and position 

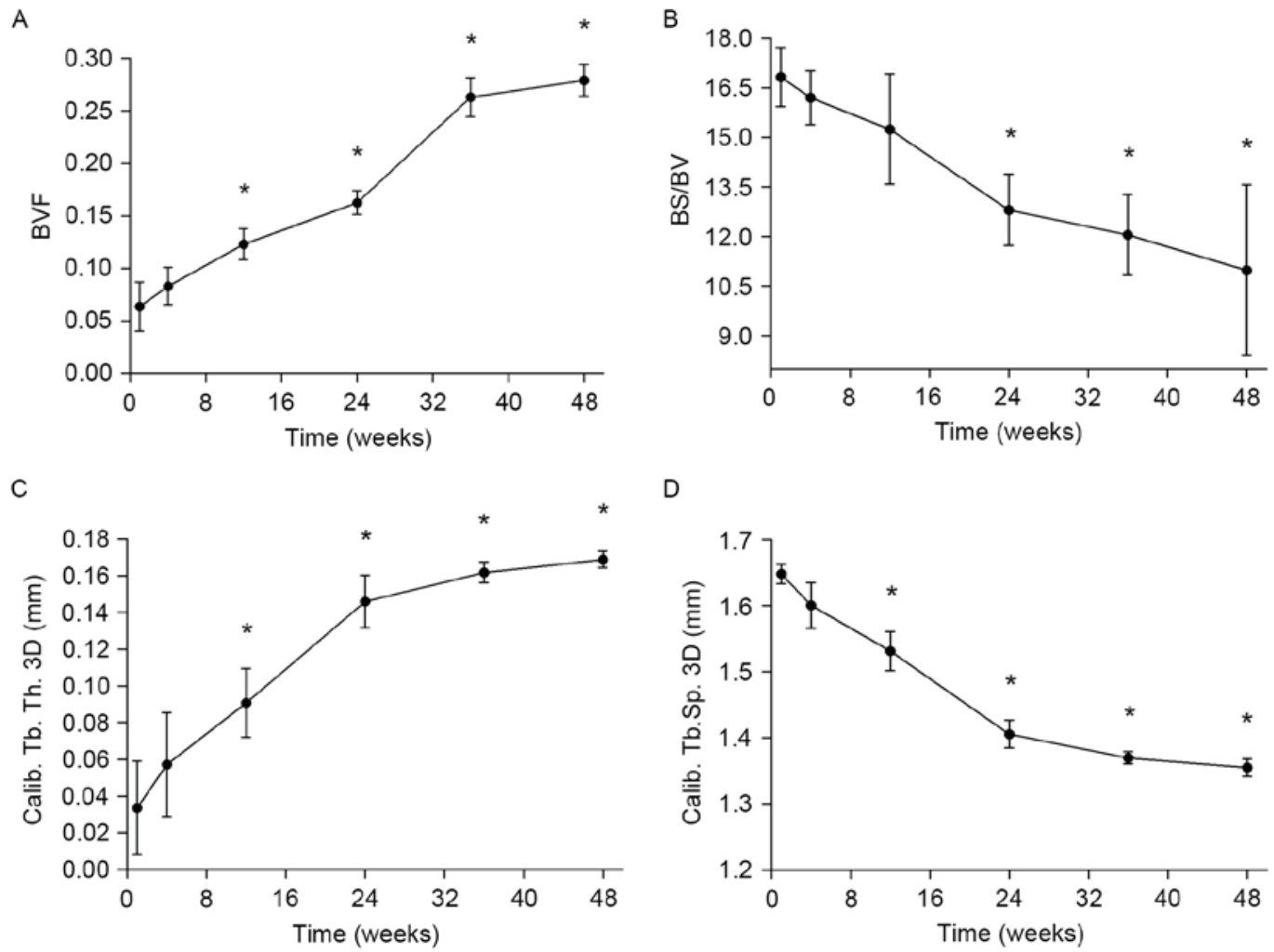

D
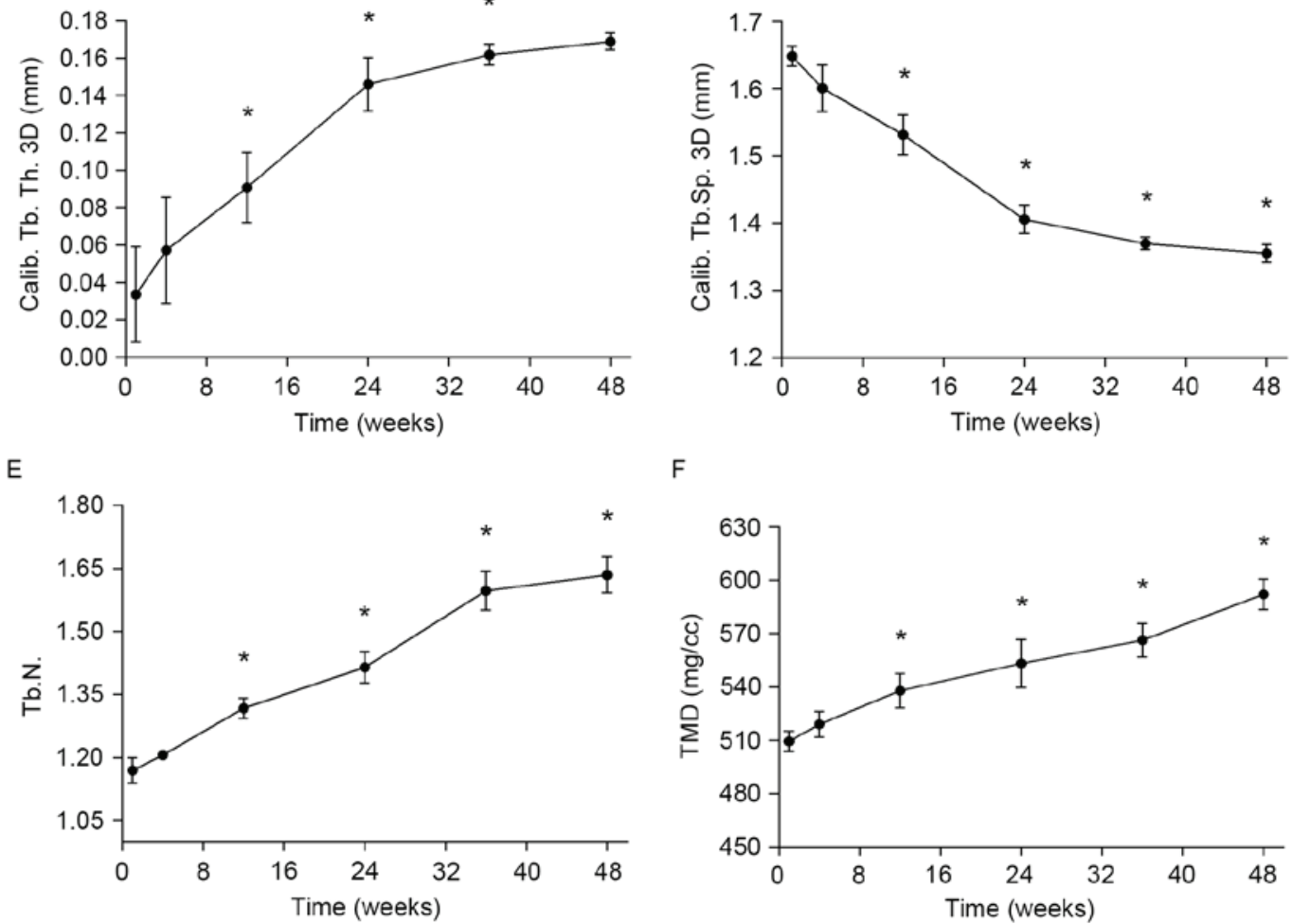

$\mathrm{F}$

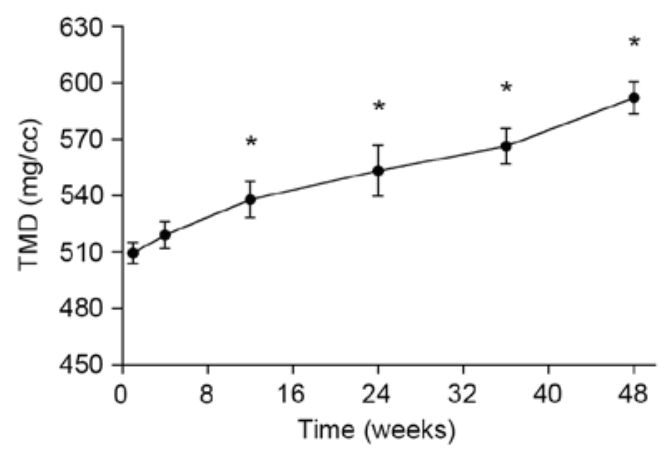

Figure 7. In vivo bone formation around the pins during the study period, assessed via micro-CT measurements. (A) BVF, (B) BS/BV. (C) Calib. Tb.Th., (D) Calib. Tb.Sp., (E) Tb.N and (F) TMD of new bone. "P<0.05 vs. prior to implantation. BVF, bone volume fraction; BS/BV, bone surface/bone volume; Calib, calibrated; Tb., trabecular; Th., thickness; Sp., separation; N., number; TMD, tissue mineral density.
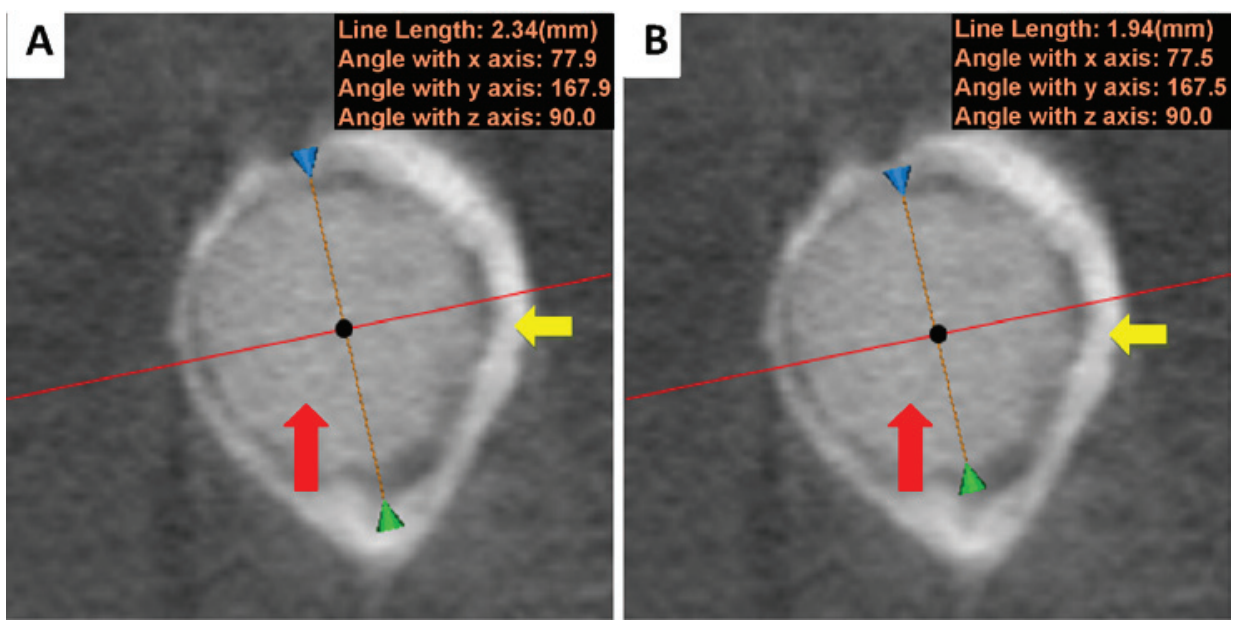

Figure 8. Micro-computed tomography images of the cross-section of the magnesium pin (week 36). (A) Maximum inner diameter of the new bone loop (orange line, $2.34 \mathrm{~mm}$ ) and (B) Diameter of the pin (orange line, $1.94 \mathrm{~mm}$ ) at the same position. Red arrows indicate the magnesium-alloy pin and the yellow arrow indicates bone formation around the pin. 
as the original ROI (Fig. 1E), from which new bone formation and the stimulatory effects on bone tissue growth were observed. The threshold was set at 1,000, and the new ROI was highlighted with the built-in software (Fig. 1F). Before week 12 , the majority of new bone formation occurred longitudinally on the surface of the metal, and the new bone appeared fragmentary and small (Fig. 6A and B). From week 4, a small amount of new bone began to grow transversely, and at the junction of the longitudinal and transverse profiles (Fig. 6B). Furthermore, the majority of new bone growth started at the bottom of the pin, which was in contact with the host cortical bone (Fig. 6B and C). From week 12, more new bone was formed, and by week 48, the pin was almost surrounded (Fig. 6C-E). The new bone growth was progressive and increased in all directions.

The data exported from the built-in software of the micro-CT equipment provided further precise results (Fig. 7). As indicated in Fig. 7A, from week 12, BVF values were significantly increased compared with the pre-implantation value $(\mathrm{P}<0.05)$. In addition, it was observed that osteogenesis increased slowly before week 24 , while from week 24 to 36 , the rate of new bone formation markedly increased (Fig. 7A). This may have been due to a faster corrosion rate and the stimulatory effects of released magnesium on the growth of new bone tissue. From week 36 to 48, the increase in BVF gradually slowed (Fig. 7A). This may be explained, to some extent, by the new bone preventing the magnesium alloy from degrading, to the disadvantage of further new bone formation. As new bone was produced, the superficial area of the ROI increased, and the ratio between the superficial area and volume of new bone (BS/BV) decreased, with significantly lower values of BS/BV observed from week $24(\mathrm{P}<0.05$; Fig. 7B). This indicates that the new bone was gradually transforming from cribrate to compact. Fig. 7C-E illustrates the thickness, separation, and number of trabecular in the new bone over time. As the pin degraded, trabecular thickness and number significantly increased and separation significantly decreased from week 12 onwards ( $\mathrm{P}<0.05$; Fig. 7C-E). Concurrently to these changes, the TMD of new bone surrounding the pin significantly increased (Fig. 7F; $\mathrm{P}<0.05$ ), indicating that the number of bones and new bone density increased as magnesium degraded.

In the cross-section of the magnesium pin, the maximum inner diameter of the new bone loop (Fig. 8A) and the diameter of the pin in the same position (Fig. 8B) were measured. During degradation, the diameter of the magnesium pin decreased. Theoretically, a firm attachment between the pin and new bone would cause the maximum inner diameter of the new bone loop to diminish, possibly faster than the diameter of the pin. As depicted in Fig. 9, the diameter of the magnesium pin decreased with degradation, from $1.98 \mathrm{~mm}$ in week 1 to $1.92 \mathrm{~mm}$ in week 48; however, no trend was observed in the maximum inner diameter of the new bone loop (max: $2.34 \mathrm{~mm}$, week 36; min: $2.08 \mathrm{~mm}$, week 1), instead of the expected downtrend. The two curves indicated that bridges had not been not adequately created by bone formation between the pin and surrounding tissues, although some bone and tissue contacts had appeared. It is probable that the biomaterials were not firmly attached to the surrounding tissues due to inadequate holding forces. This suggests that the magnesium alloy was not capable of creating sufficient

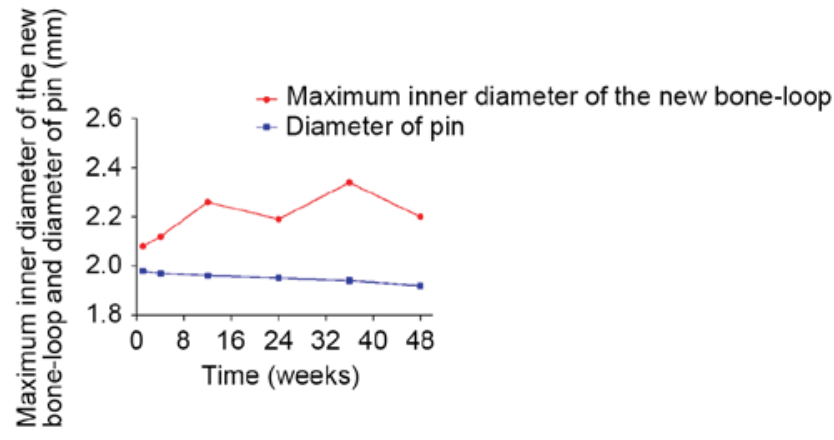

Figure 9. Maximum inner diameter of the new bone loop and diameter of the pin at the same position at each time point.

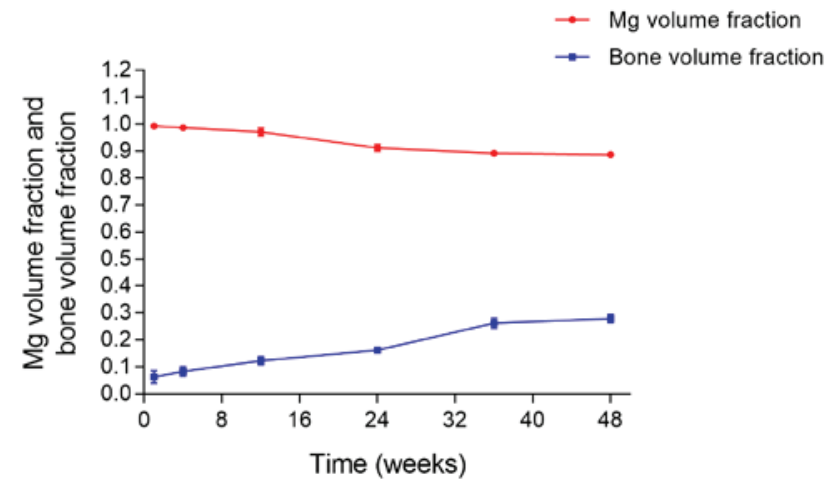

Figure 10. Pin volume fraction and bone volume fraction. At the end of the study, there was no intersection of the two curves.
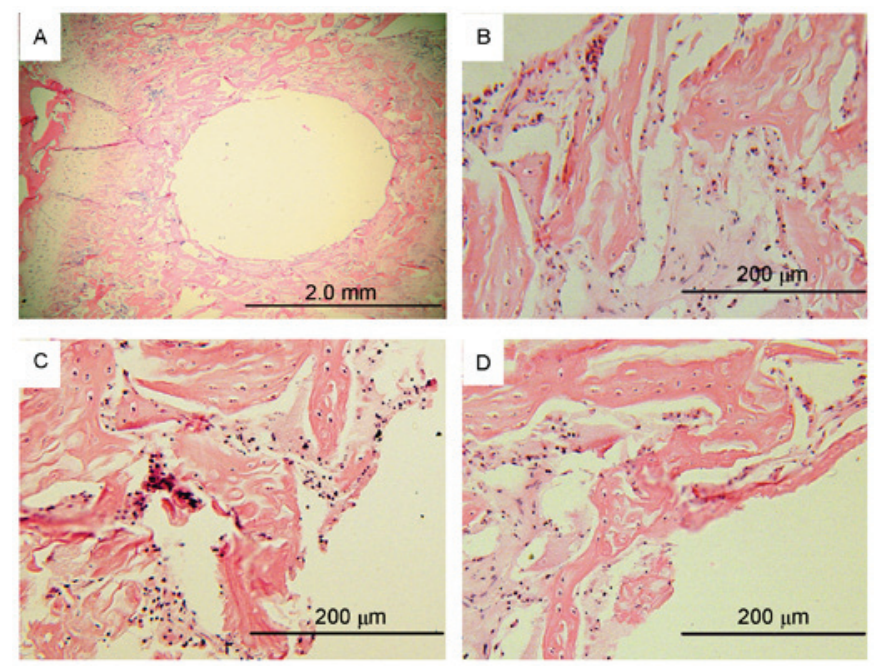

Figure 11. Hematoxylin and eosin staining of the tissue surrounding the pin (A) The hole in the middle represents the position of the pin (at 24 weeks). Tissue samples from (B) 4, (C) 24 and (D) 48 weeks.

bridges between the bones and biomaterials when there were preexisting gaps.

To investigate the association between material degradation and novel bone formation, the changes in pin volume fraction and bone volume fraction with time were plotted (Fig. 10). During the experiment, both rates were slow, and from week 1 to week 48 , the volume of the magnesium alloy at each time 
point decreased by $10.58 \%$ (from 99.23 to $88.65 \%$ ), whereas the volume of novel bone had increased by $21.54 \%$ (from 6.39 to $27.93 \%$ ). By the end of the study, there was no intersection of the two curves (Fig. 10).

Histological evaluation. H\&E staining was performed at weeks 4, 24 and 48. There were no apparent inflammatory cells surrounding the implants; however, chondrocytes and osteocytes were observed. No changes in the morphology of these cells between samples at 4, 24 and 48 weeks was observed (Fig. 11). Thus, there were no apparent inflammatory response around the implants. These findings suggested that the AZ31 magnesium alloys may be safe in human body.

\section{Discussion}

Metallic materials serve an essential role as biomaterials for the repair or replacement of diseased bone. Magnesium and its alloys have been investigated in recent years due to their advantages over other materials, such as titanium alloys and stainless steel $(57,58)$. However, due to the electrochemical activity of magnesium and its alloys in physiological solutions, it is necessary to control the rate of corrosion for safe application in the human body (59). Thus, the development of suitable strategies to enhance the corrosion resistance of magnesium alloys is important. A number of preventative measures have been proposed and are being adopted to overcome corrosion problems. While many previous studies have focused on surface-modified magnesium alloys, protective coatings must be nontoxic for orthopedic applications, and should ideally improve the biocompatibility/bioactivity of the implant $(1,3,59)$. In the present study, AZ31 magnesium (alloyed with 3 wt.\% Al, 1 wt.\% $\mathrm{Zn}$ and 0.15 wt.\% Mn) with a micro-arc surface treatment was evaluated in vivo.

The degradation of the AZ31 magnesium alloy was examined. It was demonstrated that, at 48 weeks, the total weight loss of the magnesium was only $0.058 \mathrm{~g}$, and the pin volume fraction remained at $88.66 \%$. This may have been due to the formation of new bone around the pins, decreasing the rate of degradation at later time points. In the first 4 weeks corrosion was slow, probably due to protection by the micro arc-oxidized surface, whereas during weeks 12 to 24 , the surface of the magnesium alloy may have become damaged, as indicated by an increase in corrosion. However, as the alloy degraded, it is possible that high levels of released magnesium stimulated the formation of new bone. After week 36, new bone tissue around the pins formed an enclosed space, which may have reduced contact between the pins and interstitial fluid and again slowed degradation.

The weight fraction was calculated based on the weight loss of the pins at each time point and compared with the volume fraction. The two methods used to calculate the amount of remaining pin were not wholly consistent; at each time point, the quantity of magnesium corrosion by weight was greater than by volume. Micro-CT is non-traumatic, which should improve the accuracy of the results; however, the same precision cannot be guaranteed with measurements based on weight loss. Prior to weighing, pins were isolated from the femoral condyle, dry-machined with cleaning tools, cleaned in ethanol in an ultrasonic bath and dried in warm air (23). Each of these steps may lead to a loss of material. Therefore, micro-CT was selected as the gold standard in the present study. To investigate the association between material degradation and new bone formation, the changes in pin volume fraction and bone volume fraction with time were plotted. During the experiment, both rates were slow, and at the end of week 48, the volume of the magnesium alloy had decreased by $10.58 \%$, whereas the volume of new bone had increased by $21.54 \%$. However, by the end of the study, there was no intersection of the two curves.

Magnesium exposed to a typical atmosphere will develop a gray oxide film of magnesium hydroxide $\left[\mathrm{Mg}(\mathrm{OH})_{2}\right]$, which slows further corrosion $(60,61) . \mathrm{Mg}(\mathrm{OH})_{2}$ films are slightly soluble in water; however, severe corrosion occurs in aqueous physiological environments where chloride ions are present at levels (on the order of $150 \mathrm{mmol} / \mathrm{l}$ ), as $\mathrm{Mg}(\mathrm{OH})_{2}$ reacts with $\mathrm{Cl}^{-}$to form highly soluble magnesium chloride and hydrogen gas (62). The following reactions summarize the corrosion of magnesium $(1,63)$ :

$$
\begin{aligned}
& \mathrm{Mg}_{(\mathrm{s})}+2 \mathrm{H}_{2} \mathrm{O}_{(\mathrm{aq})} \leftrightarrow \mathrm{Mg}(\mathrm{OH})_{2(\mathrm{~s})}+\mathrm{H}_{2(\mathrm{~g})} \text { (i) } \\
& \text { Equations (ii)-(iv) show the partial reactions: } \\
& \mathrm{Mg}_{(\mathrm{s})} \leftrightarrow \mathrm{Mg}^{2+}{ }_{(\mathrm{aq})}+2 \mathrm{e}^{-} \text {(anodic reaction; ii) } \\
& 2 \mathrm{H}_{2} \mathrm{O}_{(\mathrm{aq})}+2 \mathrm{e}^{-} \leftrightarrow \mathrm{H}_{2(\mathrm{~g})}+2 \mathrm{OH}^{-} \text {(aq) }(\text { cathodic reaction; iii) } \\
& \mathrm{Mg}^{2+}{ }_{\text {(aq) }}+2 \mathrm{OH}^{-}{ }_{(\mathrm{aq})} \leftrightarrow \mathrm{Mg}(\mathrm{OH})_{2(\mathrm{~s})} \text { (product formation; iv) }
\end{aligned}
$$

This hydrogenation results in an alkaline environment that raises the $\mathrm{pH}$, which is harmful to cells and decreases cell viability (64).

In the present study, implant degradation occurred at a slower rate than expected. This potentially reduced the rate of hydrogen gas production, and enabled the hydrogen to be absorbed before it accumulated (65). The micro-CT images identified a small amount of hydrogen gas around the pins at weeks 4, 12 and 48 only, and no apparent trend was observed.

The micro-CT results included both images and numerical data. The images indicated the shape of the pins and the corrosion pitting on the surface (black spots on the white surface with high signals) at each time point, and new bone formation around the pins. The numerical data indicated that the degradation of magnesium pins had undergone a 'slow-quick-slow' process, and that magnesium had stimulatory effects on the growth of new bone tissue.

Micro-CT imaging and data analysis were used to assess the consequences of degradation of micro-arc-oxidized AZ31 magnesium alloy used in bone implants. It also identified increasing amounts of new bone around the alloy during the experiment, and as the magnesium degraded, both the number of bones and new bone density increased. The micro-CT data demonstrated decreases in pin volume, mineral density, mean 'pin thickness', BS/BV, Tb.Sp, and increases in pin surface area/pin volume, BVF, Tb.Th, Tb.N and TMD, indicating a positive effect of magnesium on osteogenesis. However, the data indicated that the magnesium alloy was not capable of creating sufficient bridges between the bones and biomaterials when there were preexisting gaps. In terms of the biological safety, there were no apparent inflammatory responses around the implants. 
Overall, the results of the present study suggest that the AZ31 magnesium had a long degradation period. Further experiments should be performed to explore the degradation of AZ31 pins lacking prior surface treatment and the biomechanics of magnesium alloys.

\section{Acknowledgements}

The present study was supported by the National Science Foundation of China (grant nos. 81572148 and 51361130034), the National Key Basic Research Development Program (973 Program; grant no. 2012CB518106), the People's Liberation Army 12th Five-Year Plan Period (grant no. BWS11J025), the People's Liberation Army Key Scientific Research Program (grant no. BWS13C029) and the Beijing Science and Technology Development Foundation (grant no. Z141107004414044). The authors would like to thank the Institutes for Food and Drug Control of China, the AVIC Beijing Institute of Aeronautical Materials, Beijing Fule Science \& Technology Development Co., Ltd. and Beijing KEYU Animal Experiment Center.

\section{References}

1. Staiger M, Pietak AM, Huadmai Jand Dias G: Magnesium and its alloys as orthopedic biomaterials: A review. Biomaterials 27: 1728-1734, 2006.

2. Mantovani D and Witte F: The attraction of a lightweight metal with mechanical properties suitable for many applications brought a renewed focus on magnesium alloys in the automotive and aerospace industries. Acta Biomater 6: 1679, 2010

3. Gordon LM and Joester D: Mapping residual organics and carbonate at grain boundaries and the amorphous interphase in mouse incisor enamel. Front Physiol 6: 57, 2015.

4. Vladimirov BV, Krit BL, Lyudin VB, Morozova NV, Rossiiskaya AD, Suminov IV and Epel'feld AV: Microarc oxidation of magnesium alloys: A review. Surface Eng Applied Electrochemistry 50: 195-232, 2014.

5. Windhagen H, Radtke K, Weizbauer A, Diekmann J, Noll Y, Kreimeyer U, Schavan R, Stukenborg-Colsman C and Waizy H: Biodegradable magnesium-based screw clinically equivalent to titanium screw in hallux valgus surgery: Short term results of the first prospective, randomized, controlled clinical pilot study. Biomed Eng Online 12: 62, 2013.

6. Yamako G, Chosa E, Totoribe K, Watanabe S and Sakamoto T: Trade-off between stress shielding and initial stability on an anatomical cementless stem shortening: In-vitro biomechanical study. Med Eng Phys 37: 820-825, 2015.

7. Zielinski SM, Heetveld MJ, Bhandari M, Patka P and Van Lieshout EM; FAITH Trial Investigators: Implant removal after internal fixation of a femoral neck fracture: Effects on physical functioning. J Orthop Trauma 29: e285-e292, 2015.

8. Wong K, Yeung K, Lam J, Chu P, Luk K and Cheung K: Reduction of corrosion behavior of magnesium alloy by PCL surface treatment. Ors Ann Meeting, 2009.

9. Liu GY, Hu J, Ding ZK and Wang C: Bioactive calcium phosphate coating formed on micro-arc oxidized magnesium by chemical deposition. App Surface Sci 257: 2051-2057, 2011.

10. Kraus T, Fischerauer SF, Hänzi AC, Uggowitzer PJ, Löffler JF and Weinberg AM: Magnesium alloys for temporary implants in osteosynthesis: In vivo studies of their degradation and interaction with bone. Acta Biomater 8: 1230-1238, 2012.

11. Zhang E: Phosphate treatment of magnesium alloy implants forbiomedical applications. In: Surface Modification of Magnesium and its Alloys for Biomedical Applications. Narayanan TSNS, Park IS and Lee MH (eds). Woodhead Publishing, Waltham, MA, pp23-57, 2015.

12. Feng $\mathrm{H}$, Wang $\mathrm{G}$, Jin W, Zhang $\mathrm{X}$, Huang $\mathrm{Y}$, Gao A, Wu H, Wu G and Chu PK: Systematic study of inherent anti-bacterial properties of magnesium-based biomaterials. Acs App Mater Interfaces 8: 9662-9673, 2016.

13. Warwick ME and Binions R: Advances in thermochromic vanadium dioxide films. J Mat Chem A 2: 3275-3292, 2014.
14. Ko YM,Lee K and Kim BH: Effect of functional groups on biodegradation and pre-osteoblastic cell response on the plasma-polymerized magnesium surface. Jap J App Phy 52: 20-21, 2013.

15. Sealy MP, Guo YB, Caslaru RC, Sharkins J and Feldman D: Fatigue performance of biodegradable magnesium-calcium alloy processed by laser shock peening for orthopedic implants. Int J Fatigue 82: 428-436, 2016.

16. Sandip S, Asha K, Paulin G, Hiren S, Gagandeep S and Amit V: A comparative study of serum uric acid, calcium and magnesium in preeclampsia and normal pregnancy. J Adv Res Biol Sci 5: 55-58, 2013.

17. Yoshizawa S, Brown A, Barchowsky A and Sfeir C: Magnesium ion stimulation of bone marrow stromal cells enhances osteogenic activity, simulating the effect of magnesium alloy degradation. Acta Biomater 10: 2834-2842, 2014.

18. Yoshizawa S, Brown A, Barchowsky A and Sfeir C: Role of magnesium ions on osteogenic response in bone marrow stromal cells. Connect Tissue Res 55 (Suppl 1): S155-S159, 2014.

19. Castellani C, Lindtner RA, Hausbrandt P, Tschegg E, Stanzl-Tschegg SE, Zanoni G, Beck S and Weinberg AM: Bone-implant interface strength and osseointegration: Biodegradable magnesium alloy versus standard titanium control. Acta Biomater 7: 432-440, 2011.

20. Johnson I, Akari K and Liu H: Nanostructured hydroxyapatite/poly(lactic-co-glycolic acid) composite coating for controlling magnesium degradation in simulated body fluid. Nanotechnology 24: 375103, 2013.

21. Barfield WR, Colbath G, Desjardins JD, An, Yuehuei H, Hartsock and Langdon A: The potential of magnesium alloy use in orthopaedic surgery. Curr Orthopaedic Practice 23: 146-150, 2012.

22. Seyedraoufi ZS and Sh M: Synthesis, microstructure and mechanical properties of porous Mg-Zn scaffolds. J Mech Behav Biomed Mater 21: 1-8, 2013.

23. Fischerauer SF, Kraus T, Wu X, Tangl S, Sorantin E, Hänzi AC, Löffler JF, Uggowitzer PJ and Weinberg AM: In vivo degradation performance of micro-arc-oxidized magnesium implants: A micro-CT study in rats. Acta Biomater 9: 5411-5420, 2013.

24. Song G: Control of biodegradation of biocompatable magnesium alloys. Corrosion Sci 49: 1696-1701, 2007.

25. Chaya A, Yoshizawa S, Verdelis K, Myers N, Costello BJ, Chou DT, Pal S, Maiti S, Kumta PN and Sfeir C: In vivo study of magnesium plate and screw degradation and bone fracture healing. Acta Biomater 18: 262-269, 2015.

26. Li H, Pan H, Ning C, Tan G, Liao J and Ni G: Magnesium with micro-arc oxidation coating and polymeric membrane: An in vitro study on microenvironment. J Mater Sci Mater Med 26: 147, 2015.

27. Huang YS and Liu HW: TEM Analysis on Micro-Arc Oxide Coating on the Surface of Magnesium Alloy. J Mat Eng Perf 20: 463-467, 2011.

28. Ma WH, Liu YJ, Wang W and Zhang YZ: Improved biological performance of magnesium by micro-arc oxidation. Braz J Med Biol Res 48: 214-225, 2015.

29. Pan YK, Chen CZ, Wang DG and Yu X: Microstructure and biological properties of micro-arc oxidation coatings on ZK60 magnesium alloy. J Biomed Mater Res B Appl Biomater 100: 1574-1586, 2012.

30. Han P, Tan M, Zhang S, Ji W, Li J, Zhang X, Zhao C, Zheng Y and Chai Y: Shape and Site dependent in vivo degradation of Mg-Zn pins in rabbit femoral condyle. Int J Mol Sci 15: 2959-2970, 2014.

31. Marukawa E, Tamai M, Takahashi Y, Hatakeyama I, Sato M, Higuchi Y, Kakidachi H, Taniguchi H, Sakamoto T, Honda J, et al: Comparison of magnesium alloys and poly-l-lactide screws as degradable implants in a canine fracture model. J Biomed Mater Res B Appl Biomater 104: 1282-1289, 2016.

32. Victor SP and Muthu J: Bioactive, mechanically favorable, and biodegradable copolymer nanocomposites for orthopedic applications. Mater Sci Eng C Mater Biol Appl 39: $150-160,2014$.

33. Walker J, Shadanbaz S, Woodfield TB, Staiger MP and Dias GJ: Magnesium biomaterials for orthopedic application: A review from a biological perspective. J Biomed Mater Res B Appl Biomater 102: 1316-1331, 2014.

34. Yeung KW and Wong KH: Biodegradable metallic materials for orthopaedic implantations: A review. Technol Health Care: Sep 6, 2012 (Epub ahead of print).

35. Niu Y, Dong W, Guo H, Deng Y, Guo L, An X, He D, Wei J and Li M: Mesoporous magnesium silicate-incorporated poly ( $\varepsilon$-caprolactone)-poly(ethylene glycol)-poly( $\varepsilon$-caprolactone) bioactive composite beneficial to osteoblast behaviors. Int J Nanomedicine 9: 2665-2675, 2014. 
36. Hofstetter J, Martinelli E, Weinberg AM, Becker M, Mingler B, Peter J, Uggowitzera J and Löffler JF: Assessing the degradation performance of ultrahigh-purity magnesium in vitro, and in vivo. Corrosion Sci 91: 29-36, 2015.

37. Witkowski M, Hubert J and Mazur A: Methods of assessment of magnesium status in humans: A systematic review. Magnes Res 24: 163, 2011.

38. Schambach SJ, Bag S, Schilling L, Groden C and Brockmann MA: Application of micro-CT in small animal imaging. Methods 50: 2-13, 2010.

39. Vanderoost $\mathrm{J}$ and van Lenthe GH: From histology to micro-CT: Measuring and modeling resorption cavities and their relation to bone competence. World J Radiol 6: 643-656, 2014.

40. Karl-Göran Thorngren: Proceedings of the Swedish Orthopedic Society Helsingborg, June 1-2, 1987. Acta Orth 59: 77-100, 1988

41. Wang ZL, Yu S, Sether LA and Haughton VM: Incidence of unfused ossicles in the lumbar facet joints: CT, MR, and cryomicrotomy study. J Comput Assist Tomogr 13: 594-597, 1989.

42. Kapadia RD, Stroup GB, Badger AM, Koller B, Levin JM, Coatney RW, Dodds RA, Liang X, Lark MW and Gowen M: Applications of micro-CT and MR microscopy to study pre-clinical models of osteoporosis and osteoarthritis. Technol Health Care 6: 361-372, 1998.

43. Ding M, Odgaard A and Hvid I: Accuracy of cancellous bone volume fraction measured by micro-CT scanning. J Biomech 32 323-326, 1999.

44. Salmon P: Micro-CT 3D Image Analysis Techniques for Orthopedic Applications: Metal Implant-to-Bone Contact Surface and Porosity of Biomaterials. In: A Practical Manual For Musculoskeletal Research. World Scientific Publishing Co. Pte. Ltd., Hackensack, NJ, pp583-603, 2008.

45. Rhee Y, Hur JH, Won YY, Lim SK, Beak MH, Cui WQ, Kim KG and Kim YE: Assessment of bone quality using finite element analysis based upon micro-CT images. Clin Orthop Surg 1: 40-47, 2009.

46. Agholme F, Li X, Isaksson $\mathrm{H}, \mathrm{Ke} \mathrm{HZ}$ and Aspenberg P: Sclerostin antibody treatment enhances metaphyseal bone healing in rats. J Bone Miner Res 25: 2412-2418, 2010.

47. Wang J, Bi L, Bai JP, et al: Comparative study of micro-CT and histological section in bone morphometry. Orth J Chin: 381-384, 2009 (In Chinese).

48. Park CH, Abramson ZR, Taba M Jr, Jin Q, Chang J, Kreider JM, Goldstein SA and Giannobile WV: Three-dimensional micro-computed tomographic imaging of alveolar bone in experimental bone loss or repair. J Periodontol 78: 273-281, 2007.

49. Buie HR, Campbell GM, Klinck RJ, MacNeil JA and Boyd SK Automatic segmentation of cortical and trabecular compartments based on a dual threshold technique for in vivo micro-CT bone analysis. Bone 41: 505-515, 2007.

50. Clark DP and Badea CT: Micro-CT of rodents: State-of-the-art and future perspectives. Phys Med 30: 619-634, 2014.

51. Chang CY, Huang AJ and Palmer WE: Radiographic evaluation of hip implants. Semin Musculoskeletal Radiol 19: 12-20, 2015.

52. Ding W: Opportunities and challenges for the biodegradable magnesium alloys as next-generation biomaterials. Regen Biomater 3: 79-86, 2016.
53. Liu YJ, Yang ZY, Tan LL, Li H and Zhang YZ: An animal experimental study of porous magnesium scaffold degradation andosteogenesis. Braz J Med Biol Res 47: 715-720, 2014.

54. World Health Organization (WHO): Strategic Initiative for Developing Capacity in Ethical Review. WHO, Geneva, 2005.

55. Wong RW, Rabie B, Bendeus M and Hägg U: The effects of Rhizoma Curculiginis and Rhizoma Drynariae, extracts on bones. Chin Med 2: 13, 2007.

56. Feldkamp LA, Goldstein SA, Parfitt AM, Jesion G and Kleerekoper M: The direct examination of three-dimensional bone architecture in vitro by computed tomography. J Bone Miner Res 4: 3-11, 1989.

57. Wang J, Witte F, Xi T, Zheng Y, Yang K, Yang Y, Zhao D, Meng J, $\mathrm{Li}$ Y and Li W: Recommendation for modifying current cytotoxicity testing standards for biodegradable magnesium-based materials. Acta Biomater 21: 237-249, 2015.

58. Chen X, Geng Y and Pan F: Research progress in magnesium alloys as functional materials. Rare Metal Mat Eng 45: 2269-2274, 2016

59. Chen Y, Xu Z, Smith C and Sankar J: Recent advances on the development of magnesium alloys for biodegradable implants. Acta Biomater 10: 4561-4573, 2014.

60. Weizbauer A, Kieke M, Rahim MI, Angrisani GL, Willbold E, Diekmann J, Flörkemeier T, Windhagen H, Müller PP, Behrens P and Budde S: Magnesium-containing layered double hydroxides as orthopaedic implant coating materials An in vitro and in vivo study. J Biomed Mater Res B Appl Biomater 104: 525-531, 2016

61. Janning C, Willbold E, Vogt C, Nellesen J, Meyer-Lindenberg A, Windhagen $\mathrm{H}$, Thorey $\mathrm{F}$ and Witte F: Magnesium hydroxide temporarily enhancing osteoblast activity and decreasing the osteoclast number in peri-implant bone remodeling. Acta Biomater 6: 1861-1868, 2010.

62. Salahshoor $M$ and Guo Y: Biodegradable orthopedic magnesium-calcium ( $\mathrm{MgCa}$ ) alloys, processing, and corrosion performance. Materials (Basel) 5: 135-155, 2012.

63. Weng L and Webster TJ: Nanostructured magnesium has fewer detrimental effects on osteoblast function. Int J Nanomedicine 8: 1773-1781, 2013.

64. Hampp C, Ullmann B, Reifenrath J, Nina A, Dina D, Dirk B, Jan-Marten S and Andrea ML: Research on the biocompatibility of the new magnesium alloy LANd442-An in vivo study in the Rabbit Tibia over 26 weeks. Advanced Engineering Materials 14: B28-B37, 2012.

65. Kuhlmann J, Bartsch I, Willbold E, Schuchardt S, Holz O, Hort N, Höche D, Heineman WR and Witte F: Fast escape of hydrogen from gas cavities around corroding magnesium implants. Acta Biomater 9: 8714-8721, 2013.

(i) $\Theta$ This work is licensed under a Creative Commons Attribution-NonCommercial-NoDerivatives 4.0 International (CC BY-NC-ND 4.0) License. 